\title{
FUNCIONES PRAGMÁTICAS DE LOS RECURSOS INTENSIFICADORES EN EXPLICACIONES Y JUSTIFICACIONES ATENUANTES ${ }^{1}$
}

\author{
Javier GONZÁLEZ RIFFO \\ Pontificia Universidad Católica de Chile (Chile)
}

\section{RESUMEN}

Desde los estudios en pragmalingüística hispánica, trabajos como los de Briz y Albelda (2013) y Albelda et al. (2014) identifican en los actos de justificación y explicación un potencial carácter atenuante. Estos corresponden a estrategias que no se limitan únicamente a la presencia de una conjunción causativa, sino que también involucran un determinado contenido proposicional en el que podrían ocurrir otros fenómenos pragmáticos, como la intensificación (como en «lo hice porque te quiero mucho»). En esta línea, este trabajo pretende analizar cuáles son las funciones pragmáticas de los recursos intensificadores presentes en el contenido proposicional de explicaciones y justificaciones atenuantes en conversaciones coloquiales de hablantes de Santiago de Chile. Para esto, se realiza un análisis inductivo de cinco conversaciones coloquiales pertenecientes al corpus AMERESCO-SCL (Guerrero González y González Riffo, en línea). El análisis muestra que los intensificadores refuerzan el carácter argumentativo de las justificaciones y explicaciones y, consecuentemente, la atenuación misma. Las subfunciones que se desprenden se relacionan con las características de la argumentación que se presenta en el contenido proposicional de los recursos atenuantes en estudio.

PALABRAS CLAVE: pragmalingüistica; atenuación; intensificación; explicaciones; justificaciones.

\section{ABSTRACT}

From the Hispanic pragmatics field, publications such as Briz and Albelda (2013) and Albelda et al. (2014) identify a potential mitigating character in justification and explanation acts. These belong to strategies that are not uniquely

${ }^{1}$ Este trabajo se enmarca en el proyecto de investigación «La atenuación lingüística en el español chileno: enfoque pragmalingüístico y sociopragmático» (ANID/CONICYT Fondecyt Regular 1200003). 
limited to the presence of a causative conjunction, but also involve a determined propositional content in which other pragmatic phenomenon might occur, such as intensification (as in «I did it because I love you so much»). Continuing this line of work, this publication aims to analyze the pragmatic functions of intensifying resources found in the propositional content of the mitigating justifications and explanations in colloquial conversations of the speakers from Santiago de Chile. To this end, an inductive analysis was carried out with five audio recordings from colloquial conversations found in the AMERESCO-SCL corpus (Guerrero González and González Riffo, online). The analysis shows that intensifiers reinforce the argumentative character of justifications and explanations and, in consequence, the mitigation itself. The sub-functions found are related with the characteristics of argumentation that is present in the propositional content of the mitigating resources in study.

KEYWORDS: mitigation; intensification; explanations; justifications; pragmatics.

Fecha de recepción: 26/06/2020

Fecha de aceptación: 16/11/2020

Fecha de la versión definitiva: 24/11/2020

\section{INTRODUCCIÓN}

La atenuación pragmática ha sido definida como un recurso retóricocomunicativo que tiene por función reducir la fuerza ilocutiva de lo expresado y con ello lo que se calcula por los hablantes como amenazante o como menos exitoso (Briz y Albelda 2013). Se trata de un recurso argumentativo que se origina por necesidades de imagen social (Briz 2007). En efecto, según Albelda et al. (2014), la utilización de recursos atenuantes permite autoprotegerse de lo dicho, prevenir posibles amenazas y reparar daños a la imagen o territorio de otros. De esta forma, en una expresión como «parece que está enojado» se puede sostener que el verbo modal parece mitiga la fuerza de la aserción. Esto podría deberse a la necesidad de no comprometerse con una descripción precisa.

Por su parte, la intensificación pragmática corresponde al reforzamiento del compromiso y de las obligaciones del hablante con respecto al contenido proposicional de su enunciado (Martín Zorraquino 2004). Se trata de una operación escalar, evaluativa y que no modifica el contenido proposicional de un enunciado (Albelda 2007). En un ejemplo como «está muy enojado», el intensificador muy refuerza la evaluación del hablante, lo que implicaría un mayor compromiso epistémico con lo dicho por parte del locutor, pues este evalúa el grado de la característica enunciada, sin que el contenido proposicional se vea alterado, posiblemente porque el locutor ha calculado que el uso de esa estrategia le es afín a sus objetivos comunicativos. Así, en la interacción los recursos de atenuación e intensificación 
pragmáticas se relacionan con la imagen y territorio de los hablantes, en términos de Goffman (cf. 1959, 1967, 1974, 1981), lo que evidencia que esas descripciones se han realizado a partir del paradigma interpretativo, concretamente desde el interaccionismo simbólico.

La relación entre intensificación y atenuación también ha sido tema de discusión en la literatura hispánica, especialmente desde líneas afines al análisis conversacional. En este contexto, Schneider (2017) ofrece una revisión bibliográfica completa. Autores como Meyer-Hermann (1988) y Caffi (1999) consideran ambos fenómenos como polos opuestos de una misma escala. Desde una perspectiva conceptual distinta, Merlini Barbaresi (2009) cuestiona el carácter antinómico de lo que la autora denomina agravación.

Pese al importante desarrollo teórico que se ha evidenciado sobre esta temática, la atenuación ha captado mayor interés en trabajos empíricos que la intensificación. Precisamente desde los aportes de la Escuela de Valencia se han propuesto modelos que ofrecen taxonomías de recursos de atenuación para el español (cf. Briz y Albelda 2013; Albelda et al. 2014). Estos modelos han permitido el estudio contrastivo de diversas variedades de la lengua española, como ocurre en el proyecto PRESEEA (Proyecto para el Estudio del Español de España y América) y en estudios asociados al Grupo Val.Es.Co. En el marco de este último, también se ha estudiado la atenuación en distintos géneros discursivos. De allí que surge el interés por revisar la sensibilidad a la variación situacional (cf. Briz y Albelda 2013) y social (cf. Cestero y Albelda 2012) del uso de estos recursos. Cabe destacar que desde estos grupos y proyectos se ha realizado un número importante de trabajos teóricos y empíricos, los que han permitido profundizar en el fenómeno de la atenuación en español.

Un recurso de atenuación escasamente descrito en la literatura corresponde a las explicaciones y a las justificaciones. Al respecto, Albelda y Cestero (2011: 28) han precisado que «se puede justificar [o explicar] mediante cualquier mecanismo que apoye argumentativamente lo dicho y, a la vez, suponga una reducción del peso enunciativo del hablante». Flores y Ramírez (2015) dilucidan que las explicaciones o justificaciones se caracterizan por el ofrecimiento o aclaración de razones que restan responsabilidad a los hablantes sobre lo dicho. Por último, González Riffo y Guerrero González (2017) destacan el potencial argumentativo que tiene este recurso para respaldar la veracidad de los hechos en narraciones orales de hablantes de Santiago de Chile.

El hecho de que las explicaciones y justificaciones correspondan a un recurso discursivo y no léxico, como bien ocurre con los verbos modales, por ejemplo, posibilita que en ellas ocurran otros fenómenos lingüísticos de interés. Guerrero González et al. (2020) se centran en qué y cómo se justifica/ofrece explicaciones. En el proceso de análisis y discusión, los 
autores ponen de relieve el reiterado uso de intensificadores que se disponen en el contenido proposicional de los enunciados justificativos, como ocurre en «te lo digo porque te tengo mucho cariño", en que el segmento subrayado corresponde a la estrategia atenuadora y el ennegrecido al intensificador. Casos como estos permiten revisitar la discusión sobre la diferenciación y complementariedad entre intensificación y atenuación antes esbozada.

Esta temática de estudio es relevante, al menos, por tres razones. En primer lugar, la descripción teórica y empírica de este fenómeno particular beneficia al campo de los estudios de modalidad y subjetividad del lenguaje en general. En segundo lugar, se trata de un aporte desde el español de Chile, variedad lingüística en la que la descripción de recursos atenuantes ha sido escasa (cf. Puga 1997; González Riffo y Guerrero González 2017, 2018; González Riffo 2019). Por último, se aporta en la descripción sobre cómo los hablantes crean, recrean y negocian su imagen en la interacción. Se trata de una preocupación compartida por el análisis conversacional, por la sociolingüística interaccional y, especialmente, por los estudios sobre pragmalingüística del español.

A partir de lo señalado, en este estudio se pretende analizar la presencia de recursos intensificadores en explicaciones y justificaciones atenuantes. La pregunta que orienta la investigación corresponde a cuáles son las funciones pragmáticas de los recursos intensificadores que aparecen en explicaciones y justificaciones atenuantes en conversaciones coloquiales de hablantes de Santiago de Chile. Para dar respuesta a este interrogante, se intenta esclarecer de forma particular:

1) cuáles son los recursos intensificadores que los hablantes utilizan en sus explicaciones y justificaciones atenuantes,

2) qué rol desempeñan los intensificadores en el contenido proposicional de las explicaciones y justificaciones, y

3) cuáles son los efectos de la intensificación en el compromiso discursivo que deriva de la explicación o justificación.

Para responder estos interrogantes se realizará un análisis inductivo. El corpus que se analizará corresponde a cinco grabaciones transcritas de conversaciones coloquiales pertenecientes al proyecto AMERESCO (América y España español Coloquial) de Santiago de Chile que, en total, suman cien minutos de audio. Cada conversación es producida por dos hablantes que mantienen una relación vivencial de proximidad en un marco interaccional de familiaridad (Carcelén y Uclés 2019). 


\section{Marco conceptual Relativo a los Fenómenos de atenuación E INTENSIFICACIÓN}

\subsection{Descripción general de la atenuación y de la intensificación}

Atenuación e intensificación constituyen fenómenos pragmáticos que tienen por objeto el aumento o disminución de la fuerza ilocutiva de los actos de habla (Briz 2001). Según Bazzanella, Caffi y Sbizà (1991), los componentes graduables de la fuerza ilocutiva corresponden al contenido proposicional, la expresión de los estados internos, los roles de los interactuantes y los objetivos perlocutivos que estos persiguen en la interacción. En esta línea, atenuación e intensificación establecerían un continuum que daría cuenta de la intensidad con que un hablante comunica su mensaje. Sin embargo, y tal como Mihatsch y Albelda (2016) precisan, la determinación de la posición de un recurso particular dentro de aquella escala de intensidad es compleja, pues el contexto puede incidir de forma tal que una estrategia típicamente atenuadora se pueda utilizar de forma intensificadora o viceversa ${ }^{2}$.

Briz (2001) describe ambos fenómenos pragmáticos como acciones estratégicas, es decir, como operaciones discursivas que realizan los hablantes para alcanzar con éxito sus metas previstas. En este sentido, una situación que contraviene el cumplimiento de tales objetivos corresponde a la afección de la propia imagen o la del interlocutor. La noción de imagen ha sido ampliamente desarrollada por Goffman $(1959,1967,1974,1981)$ en sus trabajos sobre el comportamiento humano en el día a día ${ }^{3}$. Esta idea, que se manifiesta a forma de principio, media las descripciones que se hagan en torno a atenuación y a intensificación.

Albelda et al. (2014) identifican tres funciones generales de la atenuación en función de la imagen de los interlocutores. La primera de ellas corresponde a la salvaguarda del yo, en que los hablantes buscarían mejorar o, al menos, no malgastar su imagen pública. Minimizar las autoalabanzas sería una de las funciones específicas que tributan a este fin. La segunda función da cuenta de la salvaguarda del yo y del tú por medio de la prevención, como ocurre, por ejemplo, al evitar el desacuerdo. La tercera función

${ }^{2}$ Las autoras ejemplifican este caso con la ironía. En sus palabras: «la ironía actúa sobre expresiones con base semántica intensificada o atenuada e invierte su valor. Así, un uso intensificado se puede interpretar contextualmente como atenuado: La fiesta estaba repleta de gente joven podría expresar en un contexto irónico que apenas había gente joven [sic]» (Mihatsch y Albelda 2016: 11).

${ }^{3}$ La noción de imagen también ha sido desarrollada por otros autores durante los últimos veinte años (v. gr. Bravo 1999; Arundale 2006). Para una revisión detallada, véase O’Driscoll (2017). 
versa sobre la reparación de amenazas a la imagen o al territorio del otro, lo que podría presentarse al justificar los desacuerdos.

Por su parte, las funciones de la intensificación para Briz (2017) estarían relacionadas con sus dos caras: la social, vinculada con la armonía interpersonal en la interacción, y la argumentativa, que se asocia con el posicionamiento ideológico o intelectual del hablante. Asimismo, las funciones se vincularían con el carácter monológico o dialógico de la interacción. En virtud de estas consideraciones, el autor propone tres funciones de los recursos intensificadores: la (auto-) reafirmativa, la alo-reafirmativa y la contra-reafirmativa. La primera consiste en que el locutor intensifica su acción, su intención, su argumentación o su propia imagen; es decir, se trata de una intensificación del yo-hablante. La segunda tiene por objeto al interlocutor, por lo que en ella se involucra la alianza con el otro, la reafirmación del acuerdo y la cortesía. Por medio de la tercera función el hablante «refuerza argumentativamente su posición contraria en relación con el interlocutor o con terceros» (Briz 2017: 54).

En suma, la bibliografía ha descrito funciones para ambos fenómenos en virtud de sus aspectos comunicativos. De la misma forma, estas descripciones refuerzan el carácter contextual que adquieren los recursos atenuantes e intensificadores en el uso.

\subsection{Perspectivas para el estudio de la atenuación y de la intensificación}

La atenuación ha captado mayor interés en el ámbito académico que la intensificación. Concretamente, se ha estudiado la atenuación fónica (v. gr. Rojas, Blondet y Álvarez 2014; Estellés y Cabedo 2017-2018; Cabedo 2016, 2018; Hidalgo 2017) y sus vínculos con el concepto de imagen (v. gr. Albelda 2016) y con el de identidad ( $v . g r$. Kendall 2004). Asimismo, se han descrito las estrategias de atenuación en distintas lenguas y géneros ( $v$. gr. Briz y Albelda 2013; Albelda Marco et al. 2014; González Riffo y Guerrero González 2017). A propósito de estos últimos, algunos estudios se han interesado en la variación en el uso en ciertas comunidades de habla (Cestero y Albelda 2012; Flores y Ramírez 2015; Cestero 2017, Palacios 2017; Douglas, Vuoto y Soler 2018; Albelda et al. 2020, entre varios otros), y en el estudio diacrónico de la atenuación que podría posibilitar cambios lingüísticos (Samper 2018). Gran parte de los trabajos citados en el ámbito hispánico asume como trabajo seminal el de Briz (2001).

Por su parte, la intensificación se ha estudiado a partir de sus funciones (Briz 2017), de sus estrategias (Palacios y Núñez 2012; Bylinina y Sudo 2015; Moya y Carrió-Pastor 2018) y de su variación (Beltrama 2015; Enghels y Roels 2017). La investigación de ambos fenómenos pragmáticos ha evidenciado la relevancia del género discursivo y del acto de habla como factores 
que se deben considerar al determinar el carácter atenuante o intensificador de determinados recursos (cfr. Figueras 2018).

El estudio conjunto de ambos fenómenos ha sido especialmente trabajado en investigaciones con foco pragmático-discursivo en discursos de carácter jurídico, y político y jurídico. Con un interés en el primero, y desde el Análisis Crítico del Discurso, Pardo (2011) aúna ambos fenómenos (a los que denomina mitigadores y reforzadores) dentro de lo que denomina Teoría de la Tonalización, cuyo principio corresponde a que todas las lenguas jerarquizan la información con que los hablantes se expresan. En palabras de la autora, «[1] a tonalización puede metaforizarse como un sube y baja en el que siempre que algo se mitiga, algo se refuerza y viceversa. La tonalización puede observarse en un plano micro que apunta a lo gramatical y en lo macro, a lo argumentacional» (Pardo 2011: 48). En el ámbito del discurso político, Márquez Guerrero (2015) llega a la conclusión de que la atenuación es favorecida en actividades comunicativas tales como las de defenderse ante acusaciones, mientras que la intensificación se prefiere para acusar, criticar y presentar logros. Así, el uso de las estrategias de intensificación tiene por objetivo reforzar argumentativamente las posiciones de los interlocutores.

La posibilidad de abordar la atenuación y la intensificación a partir de distintas aristas permite dar cuenta de cómo la atenuación y la intensificación son sensibles al contexto situacional, social y a los fines estratégicos que los hablantes pueden perseguir en su discurso. En este trabajo, al considerar ambos fenómenos en su conjunto, también profundizamos en cómo estos construyen colaborativamente la expresión de la subjetividad ( $v . g r$. Monjour 2017).

\subsection{Sobre el carácter atenuante de las justificaciones y las explicaciones}

El carácter atenuante de las justificaciones y explicaciones se ha reconocido en varios estudios sobre la atenuación. Albelda y Cestero (2011) describen nueve tipos de procedimientos de atenuación, dentro de los cuales se halla el acto de justificación. En palabras de las autoras, «se puede justificar mediante cualquier mecanismo que apoye argumentativamente lo dicho y, a la vez, suponga una reducción del peso enunciativo del hablante» (Albelda y Cestero 2011: 26). Entre aquellos mecanismos, identifican el ofrecimiento de excusas, el empleo de comparaciones y de marcadores discursivos que presenten consecuencia lógica.

Briz y Albelda (2013) organizan las estrategias de atenuación en dos macrocategorías: la de relativizadores y la de despersonalización u ocultación. Dentro de la primera, ubican la estrategia de estructuras causales explicativas o justificativas, condicionales y concesivas. Si bien no la definen ni la describen, su adscripción a la macrocategoría de relativizadores otorga 
algunas luces. En efecto, corresponden a aquellos en que «[s]e debilita o minora la fuerza argumentativa en relación con la verdad o con la certidumbre de lo enunciado, el grado de conocimiento o el compromiso del hablante» (Briz y Albelda 2013: 304).

Albelda et al. (2014) reconocen el carácter mitigador de las construcciones justificadoras o de excusas. Sin profundizar en qué consiste este recurso, destacan que las expresiones y justificaciones pueden ser expresadas mediante partículas como es que, porque y como, o bien, sin ellas. Asimismo, incluyen dentro de esta categoría las justificaciones del decir, en que se consideran expresiones como por asi decirlo o es un decir.

Otro estudio que se ha enfocado en justificaciones y explicaciones es el de Rodríguez Alfano (2016), quien considera ambos recursos operaciones lógico-retóricas de la argumentación. Para la autora, ambas operaciones tienen en común el objetivo de «reforzar la validez de lo argumentado mediante la exposición de relaciones causales suceso-consecuencia que dan un mayor grado de certidumbre a los enunciados» (Rodríguez Alfano 2016: 168). Sin embargo, se diferencian en que las explicaciones consisten en la aclaración de la propia conducta, mientras que en la justificación se ofrecen evidencias que le permitan al hablante exonerar toda responsabilidad que le atañe por sus dichos o hechos. Desde estas premisas, la investigadora no declara que se trate de recursos atenuantes, pero sí señala que en ellas pueden ocurrir procedimientos lógico-retóricos como son la atenuación y la intensificación.

Por último, Guerrero González et al. (2020) estudian la relación entre la imagen de los hablantes y las explicaciones, justificaciones y comparaciones en actos asertivos de opinión. Los autores relevan una distinción que concierne a la actividad realizada; esto es, distinguen entre el ofrecimiento de argumentos que fundamenten una opinión y el ofrecimiento de excusas que surjan a partir de una relación natural de causa-consecuencia. Según sea el caso, variarán la imagen del hablante y el compromiso que tenga con su discurso. Siguiendo a los autores, la actividad de justificación, en general, podrá tener como función la autoprotección o la prevención, es decir, las funciones 1 o 2 dilucidadas por Albelda et al. (2014).

Debido a que en este trabajo la diferenciación entre justificaciones y explicaciones no se considera como un factor que pueda incidir en los resultados de este estudio, se utilizan ambos conceptos como equivalentes.

\section{Metodología}

Esta metodología reproduce, en gran medida, la que se utiliza para todas las investigaciones que se realizan en el proyecto de investigación 
«La atenuación lingüística en el español chileno: enfoque pragmalingüístico y sociopragmático»(ANID/CONICYT Fondecyt Regular 1200003).

\subsection{Corpus e informantes}

En este estudio se analizaron conversaciones coloquiales orales del corpus AMERESCO-SCL (Guerrero González y González Riffo, en línea). El proyecto AMERESCO pretende estudiar la variedad coloquial del español hablado de España y de América (Briz 2016) y toma como base los fundamentos metodológicos del corpus Val.Es.Co. (Briz y Grupo Val.Es.Co.).

El corpus AMERESCO se compone de registros de audio de conversaciones coloquiales, interpersonales y libres; es decir, cuyos temas no son predeterminados. En cada una de ellas participan entre dos y cuatro hablantes, considerando al investigador, quien opcionalmente podía participar de la interacción. Para este estudio, se realizó un muestreo por conveniencia que tuviese como criterio que los informantes mantuviesen al momento de la grabación una relación vivencial de proximidad y que se realizase en un marco interaccional de familiaridad para controlar factores contextuales. Con el propósito de conseguir una muestra de habla espontánea, las grabaciones fueron secretas. Todos los informantes firmaron dos consentimientos escritos previamente aprobados por los comités de ética evaluadores del proyecto MINECO FFI2016-75249P.

Si bien los informantes fueron estratificados según sus características de sexo, edad y nivel de instrucción, estos factores no se consideran como variables en esta investigación. Cada grabación fue codificada a partir de un esquema común que incluye, en orden lineal, el nombre de la ciudad abreviado con el código oficial de aeropuertos, tres dígitos para el orden interno del corpus, el número de participantes en la conversación y, por último, el año de recogida. Para cada grabación se realizó una transcripción ancha de segmentos de veinte minutos sucesivos, pues se considera que en ese lapso de tiempo se desarrolla una conversación completa (Carcelén y Uclés 2019). En este sentido, la selección de tales segmentos tuvo como criterio que en ellos se desarrollase plenamente un tema dentro de la conversación. Para la transcripción, se siguieron las convenciones Val.Es.Co. (Briz y Grupo Val.Es.Co. 2002).

El corpus de Santiago de Chile está conformado por 42 grabaciones, de características idénticas a las del proyecto general. A partir de un análisis exploratorio se determinó la conveniencia de extraer del corpus una cantidad de cinco grabaciones en que participasen como máximo dos hablantes. En la Tabla 1 se describe la muestra utilizada para este estudio: 
TABLA 1. Caracterización de la muestra

\begin{tabular}{|c|c|c|}
\hline Conversación & $\begin{array}{c}\text { Características } \\
\text { de las grabaciones }\end{array}$ & $\begin{array}{c}\text { Características } \\
\text { de los informantes }\end{array}$ \\
\hline SCL_009_02_18 & $\begin{array}{l}\text { La interacción tiene lugar } \\
\text { en una sala de estudio. } \\
\text { El tema de la conversación } \\
\text { corresponde a la necesidad } \\
\text { de organizarse como carrera, } \\
\text { además de comentar } \\
\text { cómo avanza sus proyectos } \\
\text { de organización estudiantil. } \\
\text { La grabación se realizó } \\
\text { en noviembre de } 2018 \text {. }\end{array}$ & $\begin{array}{l}\text { A y B son compañeros } \\
\text { de estudios universitarios } \\
\text { y amigos cercanos. } \\
\text { Ambos son hombres, de entre } \\
18 \text { y } 25 \text { años, y cursaban estudios } \\
\text { superiores al momento } \\
\text { de la grabación. }\end{array}$ \\
\hline SCL_015_02_19 & $\begin{array}{l}\text { La interacción tiene lugar } \\
\text { en el hogar de A. } \\
\text { Las hablantes conversan } \\
\text { sobre vacaciones y familia. } \\
\text { La grabación se realizó } \\
\text { en enero de } 2019 .\end{array}$ & $\begin{array}{l}\text { B trabaja en el hogar de A. } \\
\text { Ambas han construido una } \\
\text { relación de confianza. } \\
\text { Ambas son mujeres de entre } \\
25 \text { y } 54 \text { años. } \\
\text { A cuenta con estudios superiores } \\
\text { terminados, mientras que B } \\
\text { cuenta únicamente con estudios } \\
\text { primarios terminados. }\end{array}$ \\
\hline SCL_016_02_19 & $\begin{array}{l}\text { La interacción tiene lugar } \\
\text { en el hogar de B. } \\
\text { Los informantes conversan } \\
\text { sobre el matrimonio de B } \\
\text { y su relación con sus hijos. } \\
\text { Además, analizan las nuevas } \\
\text { generaciones. }\end{array}$ & $\begin{array}{l}\text { B es vecina de A. } \\
\text { A es mujer, mayor de } 55 \text { años } \\
\text { y de estudios primarios } \\
\text { terminados. } \\
\text { B es hombre, de entre } 25 \text { y } 55 \\
\text { años y de estudios superiores } \\
\text { terminados. }\end{array}$ \\
\hline SCL_037_02_19 & $\begin{array}{l}\text { La interacción tiene lugar en } \\
\text { el hogar de los informantes. } \\
\text { El tema de conversación } \\
\text { corresponde a la situación } \\
\text { escolar de un hijo de una } \\
\text { familia amiga de la pareja. } \\
\text { Luego, los informantes } \\
\text { conversan sobre la altura } \\
\text { de su propio hijo. } \\
\text { La grabación se realizó } \\
\text { en marzo del } 2019 .\end{array}$ & $\begin{array}{l}\text { A y B son pareja desde hace } \\
\text { más de dos décadas. } \\
\text { A es hombre, mayor de } 56 \text { años } \\
\text { y cuenta con estudios superiores } \\
\text { terminados. } \\
\text { B es mujer, mayor de } 56 \text { años } \\
\text { y cuenta con estudios } \\
\text { de secundaria terminados. }\end{array}$ \\
\hline SCL_042_02_18 & $\begin{array}{l}\text { La interacción tiene lugar en } \\
\text { el hogar de las informantes. } \\
\text { Los temas de conversación } \\
\text { corresponden a comida, } \\
\text { vida social, viajes y anécdotas } \\
\text { de ambas. }\end{array}$ & $\begin{array}{l}\text { A y B son amigas íntimas. } \\
\text { Ambas son mujeres de entre } 18 \\
\text { y } 25 \text { años. A cuenta con estudios } \\
\text { superiores terminados, mientras } \\
\text { que B realiza sus estudios } \\
\text { superiores. }\end{array}$ \\
\hline
\end{tabular}


La selección de estas cinco conversaciones cotidianas permitiría dar cuenta de varias explicaciones y justificaciones atenuantes $\mathrm{y}$, asimismo, de los recursos intensificadores que en ellas ocurren.

\subsection{Procedimiento analítico}

En primera instancia, se segmentaron los discursos en actos de habla, entendidos estos como «una unidad estructural monológica, jerárquicamente inferior a la intervención, de la que es su constituyente inmediato; asimismo, es la mínima unidad de acción e intención, que posee las propiedades de aislabilidad e identificabilidad en un contexto dado» (Grupo Val.Es.Co. 2014: 37). El acto corresponde a la unidad de análisis de este estudio, debido a que las explicaciones y justificaciones, objeto de la pesquiza, corresponden precisamente a uno.

Posteriormente, se procedió a la identificación de las explicaciones y justificaciones y se evaluó su carácter atenuante. Para esto, se consideró el contexto interaccional concreto (Briz y Albelda 2013) y conjuntamente las pruebas de conmutación, ausencia y solidaridad propuestas por Villalba (2018), las que consisten en reemplazar el atenuador por otros atenuadores o por ninguno para evaluar algún cambio en la fuerza ilocutiva; o bien verificar si se hallan otros atenuadores próximos al segmento en cuestión. En consecuencia, se analizaron las justificaciones y explicaciones que atenuasen la fuerza ilocutiva de los enunciados y que pudiesen afectar potencialmente la imagen del interlocutor.

Todas las explicaciones y justificaciones atenuantes fueron enlistadas en una planilla ordenada de parámetros de análisis que consideró las expresiones como tales, su contexto interaccional concreto y el segmento atenuado. Con esto, se dio paso a la identificación de recursos intensificadores dentro de los actos de explicación y justificación. Para la determinación del carácter intensificador de los recursos, se utilizó la taxonomía elaborada por Albelda (2007), quien clasifica los procedimientos de intensificación proposicional en recursos morfemáticos, léxicos, sintácticos, semánticos y fónicos. Para su análisis y para la posterior determinación de funciones se consideró la propuesta de Briz (2017): la (auto-)reafirmativa, la alo-reafirmativa y la contra-reafirmativa.

Por último, se realizó una caracterización con respecto a las funciones que los intensificadores cumplen en esas explicaciones y justificaciones, para lo que se consideraron los elementos que son intensificados y la relación entre el acto atenuante y lo atenuado. Las categorías que surgieron a partir de este análisis (refuerzo argumentativo, refuerzo del disenso y persuasión afectiva) fueron evaluadas repetidas veces durante todo el proceso. 
Para asegurar la solidez de los resultados y la confiabilidad de la metodología implementada, se trianguló el análisis con investigadores del proyecto AMERESCO de Santiago de Chile.

\section{RESUltados Y DiscusióN}

El análisis revela que, tal como Briz (2017) había dilucidado, el uso de recursos intensificadores se relaciona con el carácter argumentativo de las conversaciones. Esto, pues los hablantes intensifican sus puntos de vista, sus evaluaciones, en un ejercicio persuasivo que parece reforzarse ante las explicaciones y las justificaciones, debido a que ellas corresponden a recursos argumentativos. La especificidad de las funciones de los intensificadores se vincula con lo atenuado, con la justificación misma y con lo intensificado, como se desarrolla a continuación.

\subsection{Intensificación de la propia argumentación}

Ante aserciones de opinión, los hablantes suelen justificar sus posturas, debido al riesgo que la opinión puede conllevar para la imagen del opinante (Guerrero González et al. 2020). Esto puede ocurrir en contextos de clara controversia, en que los participantes de la interacción disienten entre ellos, o bien en contextos en que la controversia es potencial. El carácter atenuador de las justificaciones y explicaciones se explica precisamente por los efectos de la posible polémica en la imagen y en la armonía interpersonal en general.

En aserciones de opinión de controversia potencial, la intensificación presente en las justificaciones y explicaciones únicamente refuerza la argumentación. En este sentido, la cantidad o cualidad intensificada corresponden a respaldos de la argumentación. En (1) se puede observar dos justificaciones en que los recursos intensificadores cumplen con esta función. En el ejemplo, A y B comentan sobre la delicada situación que vive T, quien es un niño que A y B conocen:

(1) A: lamentablemente T- es lamentable la situación (2") pero tenemos que verlo como sociedad / como un grupo social y un grupo social (( )) lamentablemente hay personas que tienen que estar separadas de un- de un grupo social (2") porque se tienen que rehabilitar de una forma totalmente diferente al otro al grupo social normal porque independiente lo que el J o (e)l M que son diferentes a sus compañeros pero se comportan con sus compañeros (SCL_037_02_19; justificaciones y explicaciones subrayadas e intensificaciones ennegrecidas). 
En (1) la aserción de opinión corresponde a «hay personas que tienen que estar separadas de un grupo social», es decir, que ciertas personas deben ser excluidas de ciertos espacios de socialización. El hablante, consciente de que su juicio es controvertido y que por ello podría alentar reacciones que contravengan su imagen social, con el objetivo de salvaguardarla ofrece dos explicaciones. En la primera se intensifica la cualidad de «diferente» (forma de rehabilitación) para destacar la relevancia de la información que configura el argumento dado: es tan distinta la forma en que se debe rehabilitar que corresponde que $\mathrm{T}$ se separe del grupo social. Como se trata de un argumento de causa-consecuencia, la intensificación se produce sobre el elemento léxico en que descansa, en este caso, la causa.

Las justificaciones y explicaciones también pueden ofrecerse tras aserciones plenas, es decir, descripciones de situaciones, cosas o deseos. En estos casos, se sostiene que la imagen de los hablantes puede verse amenazada por la falta de precisión, por lo cuestionable de las descripciones o de los hechos reportados o bien porque la información que se sostiene puede amenazar la armonía interpersonal. En el ejemplo que sigue se ilustra una justificación intensificada ante una aserción plena de deseo. En (2) A y B conversan sobre locales de sushi dentro de la Región Metropolitana:

(2) B: ¿HRS? / algo así /// puta / me tinca comer es- quiero probar ese sushi que no tiene arroz

A: ¿y qué tiene?

B: todo / (RISAS) / o sea tiene como de todo los ingredientes como ricos pero como en mayor cantidaad porque hue(v)ón siento que a veces le ponen demasiado arroz hue(v)ón / no me gusta eso

(SCL_042_02_18).

En (2) B declara que desea probar un sushi que no tenga arroz. A le consulta qué tiene ese sushi si no es arroz. B responde que se reemplaza por otros ingredientes en mayor cantidad y justifica su deseo a partir de una observación crítica sobre lo que venden algunos locales. Esta justificación B la declara luego de responder a la pregunta de A, como si la pregunta de su amiga fuese también un cuestionamiento a su deseo. La evaluación final, «no me gusta eso», clarifica ese aspecto crítico que motivan los deseos de B por probar un sushi sin arroz. La justificación es atenuante porque la hablante parece ver cuestionada su intención de probar un alimento poco común y, en consecuencia, también su imagen, ante lo cual ofrece razones que argumentan su posicionamiento. La argumentación se produce sobre un juicio implícito: querer probar sushi sin arroz tiene fundamentos. La justificación, además, responsabiliza a un tercero (son ellos quienes cometen un error al preparar un sushi con mucho arroz) para proteger la propia imagen. 
La justificación cuenta con una intensificación de cantidad: «demasiado arroz». Asimismo, la hablante utiliza dos veces «huevón», estructura que parece intensificar el contenido proposicional completo del enunciado. Es interesante que no se utilice la forma femenina huevona, lo que podría reflejar que ambas ocurrencias no tienen un valor vocativo propiamente tal. El uso del cuantificador no contable en conjunto con la reiteración de «huevón» cumple con destacar la posición argumentativa de la hablante.

En segmentos narrativos, las aserciones plenas suelen tener el objetivo de relatar los acontecimientos que se reportan, pese a que en las narraciones también pueda haber aserciones de opinión. Ambas aserciones, de reporte y de opinión, pueden ser atenuadas. González Riffo y Guerrero González (2017) argumentan que las explicaciones tras aserciones plenas tienen la finalidad de argumentar la veracidad de los hechos narrados ante la posibilidad de que el o los interlocutores pongan en duda el relato. Esto último es lo que puede afectar la imagen positiva del hablante, lo que explica el carácter atenuante de las explicaciones o justificaciones que pudiera brindar.

En el ejemplo (3) A y B, ambos vecinos conversan sobre la vida de B, quien comenta algunos episodios que involucran a sus hijos. En este contexto, B ofrece algunos detalles sobre cómo esos eventos tienen relación con su vida actual. En (3) se ejemplifica uno de esos momentos en los que la hablante ofrece una explicación:

(3) B: a los treinta y ocho años [quedé viuda yo]

A: $\quad[\mathrm{uu}] /$ ¿y con cuántos hijos?

B: cinco

A: cinco / los crío usted / ¿entonces? §

B:

$\S$ si yo tengo estos lentes /

es porque yo cosía tanto a máquina y el pie de la máquina me daba / en la noche a mí / el resplendor en la vista / y eso a mí [me acortó la vista]

A: [claro]

B: pero digo yo/ ahora/ ( ( )) las mujeres se acuerdan que tienen un niño (SCL_016_02_19).

En (3), B, sin responder la pregunta que le formula A, ofrece una explicación sobre por qué usa unos lentes particulares. Su argumentación es de causa-efecto, en tanto señala que su ceguera parcial se debe a una luz que emanaba su máquina para coser, la que usaba con frecuencia. Precisamente es esto último lo que la hablante destaca por medio de una intensificación: «tanto» enfatiza la cantidad del uso por medio de un cuantificador no contable, de forma tal que la causa, en tanto tal, parece más creíble y razonable. 


\subsection{Intensificación del disenso}

El refuerzo argumentativo se puede producir en situaciones en que los interlocutores no coinciden en sus juicios. Para que esto ocurra, como condición, lo atenuado debe precisamente coincidir con la expresión de disenso. En estos casos, los intensificadores no solo refuerzan la posición del hablante, sino que también introducen y destacan su opinión. Al ser la controversia explícita en la interacción, y no una posibilidad, como ocurría en la intensificación de la propia argumentación, los intensificadores refuerzan el disenso y la argumentación torna a una contracción dialógica ${ }^{4}$.

El ejemplo (4) ilustra este caso, en que A le ha expresado a B su interés por que su hijo en común, M, practique deportes para aumentar su altura. Ante esto, B le expresa a A su desacuerdo:

(4) B: sí pero que para ti / yo lo que quiero saber pa(ra) ti (por) qué es tan importante si (él) es alto o es un poco más bajo/ porque el M tenemos súper claro que no es chico/no es bajo/ el M ya no es bajo/ porque hasta él de repente me dice yo no entiendo a mi papá ipor qué quiere que sea tan grande? (SCL_037_02_19).

En (4) B le llama la atención a A por el desmesurado interés de este último en que $\mathrm{M}$, hijo de ambos, crezca en tamaño. Ante esta situación, B declara que quiere saber a qué se debe tal interés, cuestión que atenúa por medio de dos justificaciones que permiten prevenir una potencial amenaza a su propia imagen, pues su pregunta implica cuestionar el deseo de A. Por ello, ambas justificaciones legitimarían la inquietud que tiene B. Sin embargo, en la primera justificación, B señala que M no es bajo, por lo que son contraproducentes las aspiraciones de A para que M sea más alto. Concretamente, por medio de esta justificación, B no solo ha atenuado la declaración de interés antes proferida, sino que también ha explicitado su posición ante la controversia. En este sentido, lo que se atenúa corresponde al disenso declarado indirectamente.

En este contexto, los recursos intensificadores presentes en las justificaciones podrían relacionarse con la función contra-reafirmativa propuesta por Briz (2017) y descrita en el §2.1. La primera justificación, destacada con subrayado doble, cuenta con dos instancias intensificadas: el uso de «super» ante «claro» y la repetición «no es chico - no es bajo - no es bajo». Estas instensificaciones se realizan sobre lo que sería constatable por ambos, esto es, que las evaluaciones que se puedan realizar sobre la altura de

\footnotetext{
${ }^{4}$ En términos de Martin y White (2005), la contracción dialógica concierne a la restricción del alcance de posiciones alternativas, lo que es posible realizar por medio de diversos mecanismos lingüísticos.
} 
M están supeditadas a lo que en realidad M mide, por lo que ciertas apreciaciones no son válidas ${ }^{5}$. Como ya se ha mencionado, esta justificación también explicita la posición de $\mathrm{B}$, por lo que los recursos intensificadores le son útiles para posicionar su visión como la más acertada.

En suma, ante el disenso de B, declarado indirectamente (atenuado, incluso), la hablante justifica (y, en consecuencia, atenúa) su posición para prevenir amenazas a su imagen. En sus justificaciones, B argumenta que su postura es válida y que es legítima la controversia que introduce. En este contexto, los intensificadores le sirven para reforzar su postura y, consecutivamente, el impacto que esa atenuación pueda ejercer sobre los potenciales efectos perlocutivos en su interlocutor. La segunda justificación se analiza en el siguiente apartado, pues su función concierne a una distinta a la que aquí se describe.

\subsection{Persuasión afectiva}

Como se adelantó en los ejemplos anteriores, las intensificaciones refuerzan el carácter argumentativo de las justificaciones y explicaciones atenuantes. Por esta razón, los intensificadores pueden reforzar el carácter afectivo de una información con propósitos persuasivos. Concretamente, en la segunda justificación del ejemplo (4), ahora reintegrado como (5), B introduce una tercera voz: la de M, quien no se encuentra presente en la conversación. La justificación en cuestión se ha destacado con subrayado doble.

(5) B: sí pero que para ti / yo lo que quiero saber pa(ra) ti (por) qué es tan importante si (él) es alto o es un poco más bajo/ porque el M tenemos súper claro que no es chico/ no es bajo/ el M ya no es bajo/ porque hasta él de repente me dice yo no entiendo a mi papá ipor qué quiere que sea tan grande? (SCL_037_02_19).

Su introducción se realiza por medio del adverbio «hasta», parafraseable por incluso, lo que le sirve a B de respaldo en tanto lo que ella piensa no es azaroso, sino que es compartido por otros. Es llamativo que la voz que incorpora para respaldar su posición corresponde a la del sujeto del que A y B hablan y evalúan, con el que además ambos tienen una relación afectiva. En este sentido, esta intensificación tiene por función reforzar la posición de B por medio de la inclusión de una voz que, no siendo una autoridad intelectual, obliga al interlocutor a empatizar afectivamente con ese posicionamiento.

\footnotetext{
${ }^{5}$ Nótese el uso del nosotros inclusivo en «tenemos súper claro» que incluye precisamente a su interlocutor para objetivar el juicio que B establece.
} 
En otros casos, los intensificadores se disponen en justificaciones afectivas ante aserciones de opinión que involucran personas con las que se tiene una relación íntima. En la misma conversación que (5), en (6) B comenta la situación que aqueja a J y su familia, quienes no se encuentran presentes en la interacción. A diferencia de (3), en este fragmento los interlocutores no discrepaban explícitamente en sus posiciones.

(6) yo he visto ee he hablado con ellos dos el esfuerzo el tremendo esfuerzo que ellos hacen como- / como papás- lo que- todo lo que gastan con J entonces es complicado / o sea a mí igual me- / cuando me dijo que tenían que internarlo llorando me- me complicó- / (en)tonces también teng $(\mathrm{o})$ - / pienso también cachai en que esto es bueno que esto es malo trato de decirle siempre mi punto de vista con mucha delicadeza con mucho cuidado / pero es complicado (SCL_037_02_19).

En (6) la aserción de opinión corresponde a «(lo que ellos viven) es complicado». La hablante argumenta su posición a través de la explicación que en el fragmento se visualiza subrayada. Como se anticipó, es dado el carácter afectivo presente en el recurso atenuante que la intensificación se combina con ítems léxicos que destacan precisamente tal contenido. Así, «tremendo» intensifica la cantidad de «esfuerzo» que los aludidos gastan, lo que coincide con que es precisamente esa información la que sostiene argumentalmente la opinión de B.

En síntesis, los intensificadores destacan ítems léxicos con el objetivo de reforzar la argumentación de las explicaciones y justificaciones. Como estas últimas ya corresponden per se a ejercicios argumentativos, la intensificación fortalece este proceso de forma tal que el carácter estratégicocomunicativo de la atenuación se ve reforzado, pues los hablantes dan realce a las razones que motivan sus actitudes o juicios. Si la atenuación implica una reducción del compromiso del hablante con lo dicho, el intensificar el contenido de las explicaciones y justificaciones implica lo contrario: los interlocutores se comprometen con los argumentos. La idea de Pardo (2011) en torno a que los discursos se tonalizan destacando ciertos contenidos y disminuyendo la relevancia de otros adquiere un espacio central en esta reflexión, pero ahora en las conversaciones coloquiales.

Como se ha sostenido en este trabajo, la intensificación también se caracteriza de formas distintas según el contexto en que la argumentación se desarrolla. Si bien siempre los intensificadores destacan elementos vinculados a los argumentos que se confieren, pueden hacerlo en virtud de sostener y clarificar un punto de vista (3.1), reforzar el disenso y con ello no permitir el ingreso de opiniones alternativas (3.2), o destacar elementos afectivos para persuadir al interlocutor (3.3). Si bien esta propuesta de categorización no agota todas las posibles subfunciones de los intensificadores 
en explicaciones y justificaciones atenuantes, aporta en la descripción del vínculo entre atenuación e intensificación en general.

\section{Conclusiones}

El análisis ha ilustrado que los intensificadores refuerzan el carácter argumentativo de las justificaciones y explicaciones atenuantes que ya portan ese rasgo. De esta forma, para determinar la relación entre ambos fenómenos se debe considerar no solo la información presente en las justificaciones y explicaciones, sino también lo justificado y lo explicado, en lo macro, y lo intensificado, en lo micro. Este refuerzo se concretaría en términos de los potenciales efectos perlocutivos que se persiguen con el uso de los recursos atenuantes, pues los intensificadores otorgan prominencia a la necesidad de considerar las justificaciones/explicaciones.

Concretamente, el análisis ha arrojado que la intensificación se realiza en tres aristas diferentes, las que especifican sus subfunciones. En específico, se propone que la intensificación se puede utilizar para reforzar la propia argumentación, para fundar el disenso o para reforzar la persuasión afectiva. Cualquiera que sea el caso, el recurso intensificador funciona en pro del carácter argumentativo de las justificaciones y explicaciones atenuantes. Esta intensificación, entonces, se limita a reforzar la argumentación que los hablantes ofrecen como trabajo de imagen.

Lo anterior levanta algunas inquietudes que otros estudios podrían atender. Entre ellas, destaca el efecto de contracción dialógica que pueden aportar los recursos intensificadores en el ejercicio argumentativo. Se trata de un efecto interesante que, aunque se pudo observar en pocos casos, podría complementarse con una muestra más extensa y que integre otros géneros discursivos, orales y escritos. Esto, entendiendo que la argumentación es característica de varios tipos de discursos. 


\section{BIBLIOGRAFÍA}

AlbeldA, Marta (2007): La intensificación como categoría pragmática: revisión y propuesta. Una aplicación al español coloquial, Fráncfort del Meno: Lang.

- (2016): «Sobre la incidencia de la imagen en la atenuación pragmática», Revista Internacional de Lingüistica Iberoamericana 14/1, 19-32.

- y Ana María Cestero (2011): «De nuevo, sobre los procedimientos de atenuación lingüística», Español Actual 96, 121-155.

—, Antonio Briz, Ana María Cestero, Dorota Kotwica y Cristina Villalba (2014): «Ficha metodológica para el análisis pragmático de la atenuación en corpus discursivos del español (ES.POR.ATENUACIÓN)», Oralia 17, 7-62.

—, Ana María Cestero, Silvana Guerrero y Marta SAMPER (2020): «Variación sociopragmática y geolectal en el uso de atenuación», Lengua y Habla 24, 1-53.

ARUNDALE, Robert (2006): «Face as Relational and Interactional: A Communication Framework for Research on Face, Facework, and Politeness», Journal of Politeness Research 2, 193-216.

Bazzanella, Carla, Claudia Caffi y Marina SBizÀ (1991): «Scalar Dimensions of Illocutionary Force». En Igor Zagar (ed.), Speech Act: Fiction or Reality? Proceedings of the International Conference, Ljubljana, Yugoslavia, November 15, 1991, Ljubljana: Institute za druzbene vede, 63-76.

Beltrama, Andrea (2015): «Intensification and Sociolinguistic Variation: A Corpus Study", Proceedings of the Annual Meetings of the Berkeley Linguistics Society 41, 15-30.

BRAVO, Diana (1999): «¿Imagen «positiva» vs. imagen «negativa»: pragmática sociocultural y componentes de face», Oralia 2, 155-184.

BRIZ, Antonio (2001): El español coloquial en la conversación: esbozo de pragmagramática. Barcelona: Ariel.

- (2007): «Para un análisis semántico, pragmático y sociopragmático de la cortesía atenuadora en España y América», Lingüística Española Actual 29/1, 5-40.

- (2016): «El proyecto AMERESCO. La idea de un corpus de conversaciones coloquiales del español de América». En Antonio Bañón Hernández, María del Mar Espejo Muriel, Bárbara Herrero Muñoz-Cobo y Juan Luis López Cruces (coords.), Oralidad y análisis del discurso. Homenaje a Luis Cortés, Almería: Universidad de Almería.

- (2017): «Una propuesta funcional para el análisis de la estrategia intensificadora en la conversación coloquial». En Marta Albelda y Wiltrud Mihatsch (eds.), Atenuación e intensificación en géneros discursivos, Madrid y Fráncfort: Iberoamericana/Vervuert, 43-67.

- y Marta Albelda (2013): «Una propuesta teórica y metodológica para el análisis de la atenuación lingüística en español y portugués. La base de un proyecto en común (ES.POR.ATENUACIÓN)», Onomázein 27/2, 288-319.

- y Grupo VAl.Es.Co. (2002): «Corpus de conversaciones coloquiales», Madrid: Arco/Libros. 
Bylinina, Lisa y Sudo, Yasutada (2015): «Varieties of Intensification», Natural Language \& Linguistic Theory 33/3, 881-895.

CABEdo, Adrián (2016): «The Function of Mitigation and the Prosodic Configuration: A Study from a Corpus of Colloquial Spanish», Revista Internacional de Lingüistica Iberoamericana 14/1, 55-73.

- (2018): "Atenuación con disminución prosódica significativa en géneros con distinto grado de planificación discursiva», Spanish in Context 15/2, 218-236.

CAfFI, Claudia (1999): «On Mitigation», Journal of Pragmatics 31, 881-909.

CARCELÉN, Andrea y Gloria UCLÉS (2019): «Diseño y construcción de un corpus oral multidialectal. El corpus AMERESCO», Normas 9/1, 17-36.

Cestero, Ana María (2017): «La atenuación en el habla de Madrid: Patrones sociopragmáticos», RILCE 33/1, 57-86.

- y Marta Albelda (2012): «La atenuación lingüística como fenómeno variable», Oralia $15,77-124$.

Douglas, Silvina, Joaquín Vuoto y María Amparo Soler (2018): «La atenuación en conversaciones coloquiales argentinas y españolas: un estudio contrastivo", RILCE 34/3, 1280-1312.

ENGHELs, Renata y Linde Roels (2017): «Mazo (de) y otros recursos de intensificación en el lenguaje juvenil madrileño: Factores lingüísticos y sociales», Moderna Sprak 111/2, 45-61.

Estellés, María y Adrián CABEdo (2017-2018): «La atenuación fónica en entrevistas (proyecto PRESEEA) y en conversaciones (corpus Val. Es. Co): un estudio de campo», Linred 15, 1-16.

FLoRes, Elizabeth y Gaspar RAMírez (2015): «La atenuación de los actos asertivos: diferencias entre hombres y mujeres», Sociocultural Pragmatics 3/1, 90-119.

Goffman, Erving (1959): The Presentation of Self in Everyday life, Nueva York: Anchor Books.

- (1967): Interactional Ritual: Essays on Face-To-Face Behavior, Nueva York: Pantheon Books.

- (1974): Frame Analysis: An Essay on the Organization of Experience, Nueva York: Harper and Row.

- (1981): Forms of Talk, Filadelfia: University of Pennsylvania Press.

GONZÁLEZ RIFFO, Javier (2019): «Diminutivos atenuadores en narraciones de experiencia personal de hablantes de Santiago de Chile: ¿fenómeno variable?», Cuadernos de Lingüistica del Colegio de México 6/1, 1-31.

- y Silvana Guerrero GonzÁlez (2017): «Estrategias de atenuación en narraciones conversacionales», Lengua y Habla 21, 29-44.

- y - (2018): «Recursos de atenuación en la 'orientación' de narrativas personales orales desde una perspectiva sociopragmática», Nueva revista del Pacífico $68,62-81$.

GRUPO VAL.Es.Co. (2014): «Las unidades del discurso oral. La propuesta Val.Es.Co. de segmentación de la conversación (coloquial)», Estudios de Lingüística del Español 35/1, 11-71.

Guerrero González, Silvana, Consuelo Gajardo, Javier GonzÁlez Riffo y Antonia Reyes (2020): "Lo que pasa es que la política se ha puesto farandulera": justificaciones atenuantes de aserciones de opinión en el corpus en el corpus PRESEEA de Santiago de Chile», Literatura y Lingüistica 42, 247-273. 
- y Javier GonZÁlez Riffo (en línea): «Corpus de conversaciones AmerescoSantiago de Chile». En Marta Albelda y Maria Estellés (coords.), Corpus Ameresco, <www.corpusameresco.com>, Universitat de València, ISSN: 2659-8337.

Hidalgo, Antonio (2017): «Hacia una propuesta metodológica para el estudio de la atenuación fónica en Es.Var.Atenuación», Pragmatica Sociocultural 5/1, 25-58.

Kendall, Shari (2004): «Framing Authority: Gender, Face, and Mitigation at a Radio Network», Discourse \& Society 15/1, 55-79.

MárQuez Guerrero, María (2015): «La alternancia atenuación-intensificación: estrategia de refuerzo argumentativo», Oralia 18, 185-211.

MARTIN, Jim y Peter White (2005): The Language of Evaluation: Appraisal in English, New York: Palgrave.

MARTín ZorraQuino, María Antonia (2004): «Sur l'atténuation (et l'intensification) des actes assertifs en espagnol». En Maria Helena Aráujo (ed.), Plus ou moins?! L'atténuation et l'intensification dans les langues romanes, Saint-Denis: Université Paris 8, Vincennes Saint-Denis, 247-264.

MERLINI BARBARESI, Lavinia (2009): «Linguaggio intemperante e linguaggio temperato. Ovvero intensificazione arrogante e attenuazione cortese». En Gili Fivelay Bazzanella (eds.), Fenomeni di intensità nell'italiano parlato, Firenze: Franco Cesati, 59-78.

Meyer-Hermann, Reinhard (1988): «Atenuación e intensificación (análisis pragmático de sus formas y funciones en español hablado)», Anuario de Estudios Filológicos 11, 275-290.

Minatsch, Wiltrud y Marta Albelda (2016): «Introducción. Atenuación e intensificación desde una perspectiva semántico-pragmática», Revista Internacional de Lingüistica Iberoamericana 14/1, 7-18.

Monjour, Alf (2017): “'¿Cómo que las 6:45? ¡Si a esa hora es cuando salía el tren!' ‘Se sabe algo de la reunión?, era a las 11, no?’ Usos dislocados del imperfecto español como estrategias de atenuación e intensificación». En Marta Albelda y Wiltrud Mihatsch (eds.), Atenuación e intensificación en géneros discursivos, Madrid y Fráncfort: Iberoamericana/Vervuert, 71-92.

Moya, Patricio y María Luisa CARRIó-PASTOR (2018): «Estrategias de intensificación en los comentarios digitales sobre noticias en español. Un análisis de la variación entre España y Chile», Spanish in Context 15/3, 369-391.

O’Driscoll, Jim (2017): «Face and (Im) politeness». En Jonathan Culpeper, Michael Haugh y Dániel Kádár (eds.), The Palgrave Handbook of Linguistic (Im)politeness, Londres: Palgrave Macmillan.

PALACIOS, Niktelol (2017): «Estrategias y mecanismos de atenuación en PRESEEAPuebla: Instrucción educativa baja», Anuario de Letras: Lingüistica y Filología 5, 149-182.

PALACIOS, Ignacio y Paloma NúÑEz (2012): «He's Absolutely Massive. It's a Super Day. Madonna, She is Wicked Singer. Youth Language and Intensification: A Corpus-based Study», Text \& Talk 32/6, 773-796.

PARdo, María Luisa (2011): Teoría y metodología de la investigación lingüística: método sincrónico-diacrónico de análisis lingüistico de texto, Buenos Aires: Tersites.

PugA, Juana (1997): La atenuación en el castellano de Chile. Un enfoque pragmalingüistico, Valencia: Universidad de Valencia. 
RodRíGUEz Alfano, Lidia (2016): «La atenuación en la justificación argumentativa. Un estudio aplicado al discurso de informantes con educación universitaria», Anuario de Letras. Lingüistica y Filología 4/1, 159-195.

Rojas, Darcy, María Alejandra Blondet y Alexandra Álvarez (2014): «Configuración tonal de la atenuación en el habla de Mérida», Lengua y Habla 18, 93-106.

SAMPER, Marta (2018): «Un cambio en tiempo real: La atenuación entre hablantes universitarios de Las Palmas de Gran Canaria», RILCE 34/3, 1259-1279.

SCHNEIDER, Stefan (2017): «Las dimensiones de la intensificación y de la atenuación». En Marta Albelda y Wiltrud Mihatsch (eds.), Atenuación e intensificación en géneros discursivos, Madrid y Fráncfort: Iberoamericana/Vervuert, 23-42.

Villalba, Cristina (2018): «Atenuación: algunas claves metodológicas para su análisis», Normas 8/1, 306-316. 